\title{
LA REPRODUCCIÓN DE LA VACA SE AFECTA POR LAS ALTAS TEMPERATURAS AMBIENTALES
}

\section{HIGH ENVIRONMENTAL TEMPERATURES AFFECT REPRODUCTION IN THE COW}

\author{
Agustín Góngora1', Aureliano Hernández²
}

\begin{abstract}
${ }^{1}$ MV. Dr. Sci. Grupo de Investigación en Reproducción y Genética Animal (GIRGA), Escuela de Ciencias Animales, Universidad de los Llanos. Dirección electrónica: agongora@unillanos.edu.co ${ }^{2}$ MV. PhD. Grupo de Biología de la adaptación de los animales al trópico- Facultad de Medicina Veterinaria y de Zootecnia. Universidad Nacional de Colombia.
\end{abstract}

Rev. U.D.C.A Act. \& Div. Cient. 13 (2): 141-151, 2010

\section{RESUMEN}

Se presenta una revisión crítica pertinente a los principales efectos de altas temperaturas sobre la fisiología reproductiva de la vaca, para resaltar la importancia económica y científica de su adaptación a las condiciones tropicales, en contraste con las que se presentan en países estacionales. Se altera el desarrollo folicular, el celo, la implantación, el desarrollo embrionario temprano, la gestación, el parto y el regreso al calor durante el posparto. Las pérdidas económicas ocasionadas por esta causa, no han sido estimadas en Colombia, ni en los países tropicales. Los efectos se ven más acentuados en aquellas razas que provienen del Bos taurus, dada su mayor tasa metabólica y menor capacidad para disipar el calor; sin embargo, las alteraciones que se puedan ocasionar, dependerán del grado de adaptación de los animales, lo cual, es clave para entender las excepciones existentes, en algunos casos, de razas taurinas colombianas, adaptadas a rangos de temperatura, que son nocivos en animales no adaptados. El suministro de zonas para protección de los animales de la radiación solar directa y de resguardo de las bajas temperaturas son medidas que deben evitar o disminuir el efecto del estrés térmico, por altas o bajas temperaturas. Se hace necesario reorientar los sistemas de selección genética en la búsqueda de bovinos que se adapten mejor a las condiciones climáticas propias de los ecosistemas colombianos. Las altas temperaturas causan disminución del tiempo de pastoreo y bajo consumo de alimento en la horas de mayor temperatura, lo que tiene injerencia en el desempeño reproductivo.

Palabras clave: Estrés calórico, trópico, bovinos, clima, ciclo estral.

\section{SUMMARY}

A critical review regarding the reproduction physiology of the cow, related to the main effects of high environmental temperatures, in order to point out the scientific and economic importance of its adaptation to tropical conditions, in contrast to those prevailing in temperate zones, is presented. Follicular development, estrus, embryonic implantation, gestation, parturition and return to estrus within the postpartum period are affected. However, economic losses due to heat stress have not been estimated for the Colombian cattle industry and the same appears to be true for other tropical countries. Bos taurus derived genotypes seem to be more susceptible to heat stress than the Bos indicus, given their higher metabolic rate and lesser capacity for heat dissipation. Nevertheless, alterations due to heat stress should depend on the degree of adaptation of individuals or genotypes. For instance, in Colombia there are some $B$. taurus derived breeds which are adapted to temperature ranges, deleterious to non adapted breeds. It is recommended to establish protected zones for cattle to avoid direct sun radiation exposure and protection from low temperatures, in order to avoid the correspondent effects of temperatures, above or below the range which does not affect animal physiology. It is necessary to reorient the systems of genetic selection in order to select bovines which can adapt better to different climatic conditions existing within the tropics. It should be noted that high environmental temperatures depress food consumption which then interferes in the reproductive efficiency.

Key words: Heat stress, tropics, bovines, climate, estrus. 


\section{INTRODUCCIÓN}

En el trópico colombiano prevalece gran diversidad climática, dado que se presentan altas temperaturas ambientales que sobrepasan los topes considerados como máximos, en las llamadas zonas de confort. Además, existen rangos de variación en breves lapsos (verbigracia, seis horas), durante un mismo día. Adicionalmente, en Colombia, aún permanecen ganaderías bovinas semi-intensivas y extensivas, en las que las medidas para proporcionar bienestar animal no siempre incluyen métodos que eviten la exposición a la radiación solar directa. Así mismo, la posible susceptibilidad individual o de raza que se puede presentar en los diversos genotipos utilizados en zonas del trópico bajo es un factor de considerable importancia, como causa de falla en la reproducción; por lo anterior, se consideró importante ofrecer una revisión bibliográfica del tema planteado, a la luz de la literatura disponible más pertinente.

Como animal "homeotermo", la vaca puede mantener la temperatura corporal dentro de ciertos límites fisiológicos; esta característica, le permite cumplir con las distintas funciones fisiológicas, como son su mantenimiento, la lactación y la reproducción. La adaptación de la vaca a un ecosistema específico, se puede medir por su eficiencia reproductiva; en el trópico bajo y húmedo o trópico seco existen condiciones, potencialmente adversas, que inciden en la eficiencia productiva, tales como el aumento de parásitos internos y externos, baja calidad de los forrajes, deficientes condiciones de manejo y alta temperatura y humedad relativa.

Para algunas razas, en especial aquellas que provienen del Bos taurus, aunque logran sobrevivir a las condiciones que impone el trópico, difícilmente se reproducen o su desempeño reproductivo es pobre, ocasionando serias pérdidas económicas, aun no estimadas, para muchos países tropicales. El estrés ocasionado por las condiciones climáticas tropicales húmedas o secas es el denominado estrés calórico (EC) que, en principio, induce la descarga simpático-adrenal, manifestándose en una baja producción y, que a diferencia de lo que ocurre en otras regiones del mundo, es un estrés crónico y no agudo (De Dios Vallejo, 2000; Hötzel \& Piheiro Machado, 2000).

En un estudio realizado en 2003, las pérdidas ocasionadas por EC a la industria pecuaria de los Estados Unidos, se valoraron entre 1,7 a 2,4 billones de dólares, de las que 897-1500 millones fue para la industria lechera; 370, para el ganado de carne; entre 299 a 316, para porcinos y 128 a 165 millones, para la industria avícola (St-Pierre et al. 2003). En el contexto mundial, se reconoce que más del $50 \%$ de la población bovina se ubica en los trópicos, por lo que el EC causa severas pérdidas económicas en más del $60 \%$ de la ganadería de leche (Wolfenson et al. 2000), determinando así un impacto económico global.

El EC ocurre cuando se genera un aumento en la temperatura ambiente que supera la zona termo-neutral o de bienestar para el animal (Armstrong, 1994). Dada la ubicación geográfica de Colombia (zona tropical), en donde los efectos climáticos se consideran más severos que en otras regiones, es fácil comprender que puede estar afectando la fisiología reproductiva, principalmente, en ganado de leche y de doble propósito, repercutiendo en severas pérdidas la economía ganadera.

A lo anterior, se suma la presencia, cada vez frecuente, del fenómeno del niño que, en las regiones en donde se registra, se caracteriza por un aumento en la temperatura ambiental, que supera los promedios históricos, que sumado a la tendencia del calentamiento global, amerita que sus afectos sean conocidos, analizados y comprendidos en toda su extensión.

El propósito de este artículo es describir y analizar, a la luz de la literatura científica actual, los efectos del EC sobre los eventos más importantes a nivel reproductivo, lo cual, permita la búsqueda de estrategias para incrementar la fertilidad de los animales, en las diversas regiones de Colombia.

\section{Fisiopatología del estrés calórico}

El control de la temperatura corporal es un proceso integrado, regulado por mecanismos fisiológicos que tienen un punto central en el núcleo ventro-medial del hipotálamo. Los efectos más severos del EC, se observan en la leche de vaca, dado que es poco eficiente en mantener la temperatura corporal, debido a que la pérdida de calor depende, esencialmente, de la evaporación por vía respiratoria y, en menor grado, por la sudoración (Patton,1994). La temperatura y el movimiento del aire, la humedad relativa, y la radiación solar son las variables que mayor influencia tienen sobre la temperatura ambiente (Buffington et al. 1981). Bajo condiciones controladas en vacas Holstein, Johnson et al. (1961) establecieron que a determinados valores de temperatura y humedad se empezaba a disminuir la producción de leche, a la vez, la estrecha asociación entre estas dos variables permitió establecer un índice de temperatura-humedad (ITH) y un valor crítico de 72, valor por encima, del cual, se empieza a afectar la producción de leche.

Se identificó una zona termo-neutral o de confort para el animal, en donde no se observan efectos sobre la disipación de calor, ni alteraciones en las funciones fisiológicas, metabólicas y productivas, por consiguiente, la expresión del potencial 
productivo es máximo (Fraser $\mathcal{E}$ Broom, 1990). La zona termo-neutral para ganado de leche, se encuentra entre 5 y $21^{\circ} \mathrm{C}$, aunque dentro de este rango, se ha propuesto otro más estrecho entre 5 y $18^{\circ} \mathrm{C}$ (Shearer $\mathcal{E}$ Bray, 1995). En Colombia, estas zonas se ubican, principalmente, en los altiplanos fríos y de clima medio. Para ganado de carne, la zona termo-neutral se halla entre 4 a $27^{\circ} \mathrm{C}$ (Yousef, 1985), que corresponde, en nuestro país, a la zona de trópico bajo, húmedo o seco.

Como respuesta a los cambios ambientales, el animal disminuye la producción de calor y, por consiguiente, aumenta el intercambio con el ambiente externo, busca la sombra, disminuye la actividad física y el consumo de alimento, lo que se traduce en una baja producción de leche, hasta en un 25 a 30\% (Maust et al. 1972). Durante el tiempo que transcurre y que conlleva a la adaptación del animal frente al EC, se comprometen otros sistemas fisiológicos de importancia (Aréchiga-Flórez, 2000).

Cuantitativamente, la pérdida de calor interno mediante el sudor es la más importante en las razas cebuínas; en animales que se mantuvieron a $40^{\circ} \mathrm{C}$, el $84 \%$ del calor total fue eliminado por esta vía (Robertshaw, 1984). A pesar del intento por seleccionar animales resistentes al EC, estudios adelantados en Australia, demostraron que la heredabilidad para esta característica es de baja a moderada (Mackinnon et al. 1991; Burrow, 2001).

\section{Efectos del estrés por calor sobre el ciclo estral}

El EC altera la intensidad y la duración del estro, el cual puede disminuir en cinco horas, respecto al promedio para algunas regiones templadas (11,9 horas) (Badinga et al. 1985). Igualmente, se afecta el desarrollo folicular y la fuente preovulatoria de LH, lo que favorecería un retardo en la ovulación o que ésta no se presente (Moberg, 1975; Gwazdauskas et al. 1981). Todavía no se conoce, con exactitud, el momento de mayor susceptibilidad al EC antes de la ovulación (Hansen, 2007); sin embargo, Al-Katanani et al. (1999) encontraron una asociación entre las altas temperaturas el día diez antes de la ovulación, con una reducida fertilidad después de la inseminación. En otro estudio, se percibió efectos desde el día 50 y 20 antes de la ovulación sobre la tasa de preñez (Chebel et al. 2004)

Nebel et al. (1997) observaron en vacas Holstein en estro, una disminución de la actividad sexual en el verano frente al invierno (4,5 montas y 8,6 montas, respectivamente); este comportamiento redujo la probabilidad de su detección por observadores entrenados. El porcentaje de celos no detectados en fincas comerciales en Florida (EU) estuvieron entre 76 y $82 \%$ de junio a septiembre y entre 44 y $65 \%$, entre octubre a mayo (Thatcher $\mathcal{E}$ Collier, 1986). Se ha contemplado que la principal razón por la que se reduce la expresión del estro es la inactividad física del animal, que busca disminuir la producción de calor evitando así la detección por los observadores (Aréchiga-Flórez \& Hansen, 2003).

El EC altera el desarrollo y la dominancia folicular durante los primeros ocho días del ciclo estral. Si este efecto se mantiene en forma crónica, la actividad de la aromatasa y las concentraciones de estradiol $\left(\mathrm{E}_{2}\right)$ en el líquido folicular disminuyen (Badinga et al. 1993). Después de la ovulación, se afecta la producción de progesterona (P4) por el cuerpo lúteo (CL), se modifica el micro ambiente del oviducto y del útero, lo que compromete la sobrevivencia del embrión (Breuel et al. 1993).

Wolfenson et al. (1995) encontraron que el EC durante los meses de verano, en los países de la zona templada, afecta la emergencia de las ondas de crecimiento folicular en aquellas vacas que presentaron dos ondas; dichos efectos fueron asociados por otros autores, con la infertilidad temporal (Thatcher, 1996; Wolfenson et al. 1997). En novillas Holstein afectadas por el EC, entre los días 11 a 21 del ciclo estral, se evidenció un menor tamaño de los folículos y menores concentraciones de $\mathrm{E}_{2}$; aunque los niveles de $\mathrm{P}_{4}$ fueron normales, ocurrió un retardo en la luteólisis y la emergencia de una nueva onda, favoreciendo la aparición de ciclos estrales, con tres ondas foliculares (Wilson et al. 1998).

En vacas Gyr no se encontraron efectos inmediatos del EC sobre la actividad reproductiva, los cuales, fueron evidentes, posteriormente, sobre el desarrollo folicular, producción de hormonas y capacidad de desarrollo del oocito (TorresJúnior et al. 2008). Estos efectos ya habían sido estudiados con anterioridad a partir de oocitos obtenidos por aspiración folicular sin que alcanzaran el estado de blastocito; la restauración normal, en la calidad de los mismos, aconteció solamente después de seis ondas foliculares o el equivalente a tres ciclos estrales (Roth et al. 2001).

El EC por un período de diez horas el día del celo, afectó negativamente la calidad del oocito, el desarrollo del embrión y su sobrevivencia (Thatcher et al. 1993). Recientemente, el menor desarrollo de los oocitos no fue atribuido a las concentraciones de Oxígeno, $\mathrm{E}_{2}$ y $\mathrm{P}_{4}$, por lo que otras moléculas o mecanismos deben ser investigadas (De Castro e Paula et al. 2008).

Se ha establecido que la susceptibilidad al EC en los estados tempranos de desarrollo embrionario, se debe a la ausencia de proteínas de "shock calórico", producidas por el propio embrión; sin embargo, a medida que avanza su desarrollo, logra elaborarlas, lo que le brinda protección (Bañuelos et 
al. 2001). Este grupo de proteínas actúan como chaperonas y cumplen importantes funciones biológicas, como el plegamiento y la síntesis de otras proteínas, favorecen, a la vez, su paso a través de las membranas en los diferentes compartimentos celulares, como el retículo endoplásmico y la mitocondria, previniendo su desnaturalización (Ju, 2005).

No solo las altas temperaturas afectan la función reproductiva y el comportamiento sexual, sino también la lluvia excesiva (Plasse, 1994). En zonas tropicales, la caída repentina de la temperatura tuvo un efecto negativo en el ganado Cebú (Plasse et al. 1968; 1970). En Bolivia, en la región del Beni, las vacas Cebú disminuyeron su actividad sexual frente a las vacas criollas o cruzadas, como consecuencia de los vientos fríos del sur, que ocasionaron un descenso de la temperatura entre 7 a $14^{\circ} \mathrm{C}$ (por debajo de la zona termo-neutral para la raza). Se requiere conocer si esta misma situación se registra en algunas regiones de los Llanos orientales de Colombia, en el ganado Cebú, en donde es notoria la influencia de los vientos fríos, provenientes de Brasil, durante el invierno en ese país.

En un estudio realizado por los autores en novillas Brahmán y Sanmartineras, en la región del Piedemonte Llanero, no se apreciaron diferencias en la duración del celo y el ciclo estral entre épocas (lluvia y sequía) (Góngora \& Hernández, 2006).

\section{Efectos del estrés calórico sobre el desarrollo embrionario}

La elevada temperatura ambiental conlleva un aumento de la temperatura uterina, que incrementa la mortalidad embrionaria (ME). Cuando la temperatura rectal aumentó de 38,5 a $40^{\circ} \mathrm{C}$, por 72 horas posinseminación, las tasas de preñez cayeron de 48 a 0\% (Dunlap \& Vincent, 1971). Estudios efectuados en Florida (EU) demostraron que un aumento de $0,5^{\circ} \mathrm{C}$ en la temperatura vaginal el día del celo o un día después ocasionó disminución en las tasas de preñez. Cuando las temperaturas rectales aumentaron en $1^{\circ} \mathrm{C}$, las tasas de preñez cayeron $16 \%$ (Ulberg \& Burfening, 1967).

En novillas Holstein super-ovuladas y expuestas a altas temperaturas, solo el 20,7\% de los embriones recuperados fueron normales (Putney et al. 1988). Se hace necesario, a este respecto, identificar en el país las épocas más favorables, en donde se podrían concentrar el mayor número de transferencias embrionarias, lo cual, sería válido para los programas de inseminación a tiempo fijo.

Aunque en el país no se tienen estudios sobre los efectos del fenómeno del Niño, en vacas donantes de embriones se encontró una asociación entre este fenómeno, con una reducción del número de embriones, la menor calidad y viabilidad de los mismos (Benyei et al. 2003).
Se ha observado un aumento en la ME antes del día siete de la gestación (Monty $\mathcal{E}$ Racowsky, 1987), de allí que la transferencia de embriones (TE) sería de utilidad en disminuir el efecto del EC, ya que los embriones se transfieren entre los días 6 a 8, cuando son más resistentes al EC (Rutledge, 2001).

Putney et al. (1989) observaron durante el verano, que las tasas de concepción de vacas lactantes receptoras era mayor (29\%) que las vacas inseminadas artificialmente (13\%), lo que confirma la utilidad de la transferencia embrionaria, para mejorar la fertilidad, en épocas de verano.

En vacas de razas usadas para producción de carne expuestas al EC, entre los días 8-16 de la gestación, se obtuvo una disminución en el peso del conceptus y del CL (Biggers et al. 1987). La ME asociada al EC entre los días 7-14 pudo ser explicada por una falla en la secreción de Interferón Tau (Geisert et al. 1998), considerado la señal de reconocimiento materno de la preñez. Los embriones de ganado adaptado al trópico (Brahman) son más resistentes al EC que los de ganado Holstein (no adaptado) (Krininger et al. 2003). Igual situación se observó en embriones de ganado Romosinuano y Brahman, los cuales, fueron más resistentes al EC que los embriones de ganado Holstein y Angus, lo que sugiere, que el periodo de adaptación al trópico ha permitido la selección de genes que controlan la tolerancia de las células al calor (Hernández-Cerón et al. 2004).

Es posible que las altas temperaturas causen disminución en el volumen de los fluidos amniótico y alantoideo, en embriones de 30 días de edad (Lee, 1993). Algunas investigaciones, se orientaron a disminuir el efecto de las altas temperaturas sobre las células del embrión, tratando de modificarlo bioquímicamente, evitando el efecto de los radicales libres. La adición de antioxidantes al medio de cultivo que contenía embriones expuestos a temperaturas elevadas, podría incrementar la viabilidad embrionaria (Hansen $\mathcal{E}$ Aréchiga, 1999); no obstante, recientemente, una revisión llevada a cabo por Hansen (2007) sobre el tema, no se han encontrado efectos de la administración de Selenio, Selenio más vitamina E y $\beta$-caroteno.

$\mathrm{Ju}$ (2005), en oocitos bovinos in vitro sometidos a $42^{\circ} \mathrm{C}$, observó alteraciones en el citoesqueleto, aunque no se evidenciaron diferencias en las tasa de clivaje; hubo una disminución notoria del número de células del blastocito y del trofoectodermo, además de alteraciones en la formación del huso meiótico. En otros dos estudios in vitro, los oocitos sometidos a $41^{\circ} \mathrm{C}$ fue evidente una reducción en la maduración nuclear y un aumento de pronúcleos apoptoticos (Roth E Hansen, 2004; 2005). 


\section{Efectos del EC sobre la gestación, parto y posparto}

Los efectos del EC sobre la preñez, se relacionan con la disminución del peso del ternero al nacimiento, alteración de las concentraciones hormonales de la madre y del feto y reducción en la producción de leche durante el posparto (Collier et al. 1982). La reducción en el peso del ternero fue $18 \%$ y se atribuyó a una disminución en las concentraciones de sulfato de estrona.

En otro estudio, se observó un menor flujo sanguíneo uterino y umbilical, un menor peso del hígado fetal y menor contenido total de RNA y proteína (Reynolds et al. 1985). También se podría afectar el desarrollo vascular de la placenta (Head et al. 1981), proceso que es crítico en la supervivencia embrionaria, pues el proceso de vasculogénesis en la membrana corioalantoidea en los rumiantes es un proceso lento (Jiménez E Hernández, 1982; Rivas et al. 2006) y la evaluación de la mortalidad embrionaria post-mortem, se basa, entre otros aspectos, en la extensión del área vascular alantoidea (Rodríguez et al. 2005).

Bajo intensa radiación solar, alta humedad y temperatura, el animal puede retardar o suspender el proceso del parto (Shearer et al. 1996). Se halló, además, una mayor concentración sérica de inmunoglobulinas (Igs) en terneros de 2-10 días, en el invierno (Mohammed et al. 1991) y una disminución, durante el verano, debido a la mayor concentración de corticoides séricos, que pueden reducir la permeabilidad intestinal de las Igs o la respuesta natural al amamantamiento, que coincidió con los picos de calor y estrés ambiental (Stott et al. 1976; Shearer, 1992).

Los efectos de la hipertermia en el último tercio de la gestación podrían estar asociados con elevadas concentraciones de 13-14 dihidro-15keto prostaglandina, durante el posparto temprano, las cuales, posiblemente, incrementan el proceso de involución uterina, sin alterar el primer estro posparto, el número de días abiertos, ni el número de servicios por concepción (Collier et al. 1982). En un estudio reciente en vacas Holstein, se evaluaron los efectos del EC sobre la actividad luteal posparto y consecuente desempeño reproductivo, donde se registró una alta actividad luteal anormal (retardo en la ciclicidad y anovulación), durante la estación seca, acompañada de una caída en la condición corporal a la quinta semana, aunque no fueron claros los efectos sobre la involución uterina (Kornmatitsuk et al. 2008).

En vacas Holstein estresadas por calor, el balance energético negativo y el aumento en los días abiertos fue asociado con una disminución del apetito e ingestión de materia seca, lo que contribuyó a un incremento del intervalo parto-concepción. A este respecto, se hace necesario delimitar los efectos ocasionados estrictamente por el EC, con los generados por causas nutricionales, ya que en la mayoría de los casos, se encuentran asociados (Ashworth et al. 2009).

El EC en el posparto temprano afectó el balance energético negativo, la condición corporal, el diámetro del folículo dominante y las concentraciones bioquímicas del líquido folicular, dando lugar a una disminución de la calidad del oocito y de las células de la granulosa, que conllevaron una baja fertilidad (Shehab-el-deen et al. 2010)

Con referencia a otras aspectos en la salud, se encontró una mayor incidencia de infecciones de la ubre y aumento en la frecuencia de mastitis en los meses de verano, lo que sugiere que, en esta época, los mecanismos de defensa de la glándula mamaria podrían estar disminuidos (Giesecke, 1985). En la tabla 1, se resumen los principales efectos del EC sobre la reproducción y la respectiva fuente bibliográfica.

\section{Alternativas para disminuir el impacto del EC sobre la reproducción}

Se han planteado diversas estrategias para reducir la magnitud del EC, entre ellas, la modificación del medioambiente, las de tipo genético, con el objeto de seleccionar animales con mayor capacidad de regular la temperatura corporal, las nutricionales, las de manejo y las farmacológicas; sin embargo, la más económica continúa siendo el suministro de sombra.

La implementación de sitios de sombrío empleando árboles es un proceso que puede tardar años, por lo tanto, el humedecimiento de la piel de las vacas, en ciertas horas del día (enfriamiento), es una manera efectiva para evitar la hipertermia, aunque lo ideal sería una suficiente disponibilidad de fuentes de agua, para el baño del ganado ad libitum.

En Israel, se comparó el efecto de la sombra más la aplicación de baños y de ventilación, cada tres horas, durante el momento de máxima temperatura (al medio día), frente a vacas control. En el grupo experimental, hubo aumento de la duración del estro y la tasa de fertilidad, después de la inseminación artificial. Dicho efecto, se observó al primer servicio y a los 150 días. El mismo tratamiento aplicado a las vacas al final de la gestación, aumentó el peso del ternero al nacimiento y la producción de leche. En Irak, la utilización de la misma estrategia disminuyó el intervalo entre partos $(384,1$ vs 376 días) y el número de inseminaciones por preñez (1,82 vs 1,73$)$ (Jassim \& Ray, 1986). 
Tabla 1. Efectos del EC sobre los diferentes mecanismos fisiológicos reproductivos.

\begin{tabular}{|c|c|c|}
\hline PROCESO FISIOLÓGICO & MECANISMO AFECTADO & REFERENCIA \\
\hline Celo & Intensidad y duración del celo & $\begin{array}{l}\text { Badinga et al. 1985; Hansen \& Arechiga, } \\
1999\end{array}$ \\
\hline \multirow{3}{*}{ Desarrollo folicular } & Dominancia folicular, esteroidogénesis folicular & Badinga et al. 1993 \\
\hline & Tamaño de folículos y concentraciones de $\mathrm{E}_{2}$ & Wilson et al. 1998 \\
\hline & $\begin{array}{l}\text { Efectos retardados sobre desarrollo folicular, producción de } \\
\text { hormonas y capacidad de desarrollo del oocito }\end{array}$ & Torres-Júnior et al. 2008 \\
\hline \multirow[t]{2}{*}{ Ovulación } & Retardo o supresión de la ovulación & $\begin{array}{l}\text { Moberg, 1975; Hansen, 2007; Al-Katanani et } \\
\text { al. 1999; Chebel et al. } 2004\end{array}$ \\
\hline & Secreción pre-ovulatoria de LH & Gwazdauskas et al. 1981 \\
\hline \multirow{3}{*}{ Integridad del oocito } & Alteración del citoesqueleto y huso meiotico & Ju, 2005 \\
\hline & Maduración nuclear & Roth \& Hansen, 2004 \\
\hline & Maduración nuclear y aumento apoptosis & Roth \& Hansen, 2005 \\
\hline \multirow[t]{2}{*}{ Desarrollo embrionario } & Sobrevivencia embrionaria temprana & $\begin{array}{c}\text { Dunlap \& Vincent, 1971; Biggers et al. 1987; } \\
\text { Putney et al. } 1988\end{array}$ \\
\hline & Secreción de Interferón Tau & Geisert et al. 1998 \\
\hline \multirow{3}{*}{ Gestación } & Desarrollo vascular de la placenta & Head et al. 1981 \\
\hline & Flujo sanguíneo al feto & Reynolds et al. 1985 \\
\hline & Desarrollo fetal & Collier et al. 1982 \\
\hline Parto & Retardo o suspensión del parto & Shearer et al. 1996 \\
\hline \multirow{2}{*}{ Posparto } & Ciclicidad y ovulación posparto & Kornmatitsuk et al. 2008 \\
\hline & $\begin{array}{c}\text { Balance energético, condición corporal y concentración de } \\
\text { hormonas en líquido folicular }\end{array}$ & Shehab-el-deen et al. 2010 \\
\hline
\end{tabular}

Los sistemas silvopastoriles podrían aliviar los efectos del EC. En Costa Rica, en vacas Brahman x Pardo suizo, para evaluar el efecto de la sombra sobre el comportamiento animal, se evidenció un mayor tiempo de pastoreo y una considerable producción de leche, en el grupo que pastaba en potreros, con adecuada cantidad de árboles, en comparación con las vacas control, que se mantenían en praderas sin sombra (Betancourt et al. 2005).

El desarrollo de nuevas razas, se viene implementando en algunos países; tal es el caso de la raza Senepol, en la que se ha identificado un gen que controla la longitud del pelo, lo que incrementa la capacidad para regular la temperatura corporal frente al EC (Olson et al. 1997). Respecto al uso de distintos cruzamientos, Pegorer et al. (2007), durante el verano en
Brasil, registraron un aumento de las tasas de preñez en vacas Holstein lactantes cuando fueron inseminadas con semen de toros Gyr versus toros Holstein; estos resultados fueron atribuidos al efecto de la heterosis del embrión (Boediono et al. 2003).

Se ha planteado una estrategia farmacológica utilizando somatotropina recombinante bovina (bST) para aliviar los efectos del EC, aunque los resultados son contradictorios (Caballero et al. 1995). En un estudio, la bST aumentó la severidad de la respuesta de las vacas estresadas por calor sin obtener cambios en la producción de leche (Cole \& Hansen, 1993), mientras que en Israel, se obtuvo un aumento del $25 \%$ en la producción total de leche, $15 \%$ en grasa y $13 \%$ en la producción de proteínas totales (Lotan et al. 1993). 


\section{CONCLUSIONES Y PERSPECTIVAS DE INVESTIGACIÓN}

El EC puede ser una causa importante de infertilidad bovina en diferentes regiones naturales de Colombia, caracterizada por una gran diversidad climática; sin embargo, no existen estudios que demuestren el verdadero impacto económico sobre la producción.

El suministro de zonas para protección de los animales de la radiación solar directa y de zonas para protección de las bajas temperaturas son medidas que deben evitar o disminuir el efecto del estrés térmico, por altas o bajas temperaturas ambientales.

Se requiere el inicio de estudios inmediatos sobre el resto de las razas criollas, tendientes a demostrar, tal como ha ocurrido con el Romosinuano, su resistencia al EC (Hammond et al. 1996). Es posible que la permanencia por más de 500 años a las difíciles condiciones que impone el trópico haya ocasionado cambios en la fisiología y, entre ellos, los mecanismos de resistencia al estrés por calor; esto llevaría a reorientar los programas de cruzamiento, teniendo como base este recurso. Iguales estudios, se deberían emprender con el resto de las razas criollas.

La TE sería de utilidad práctica en mejorar la fertilidad en épocas de verano, lo cual, podría ser acompañado de protocolos de inseminación artificial a tiempo fijo, evitándose la necesidad de detección del estro. Concomitantemente, se hace necesario identificar las épocas más favorables para los procedimientos de aspiración folicular y producción de embriones in vitro, pues tal como ha sido evidenciado en el presente artículo, aquellas épocas de alta temperatura ambiental tiene efectos deletéreos sobre el oocito, independiente del genotipo del animal.

Conflicto de intereses: El manuscrito fue preparado y revisado por los autores, quienes declaran que no existe ningún conflicto de intereses.

\section{BIBLIOGRAFÍA}

1. AL-KATANANI, Y.M.; WEBB, D.W.; HANSEN, P.J. 1999. Factors affecting seasonal variation in 90 day non-return rate to first service in lactating Holstein cows in a hot climate. J. Dairy Sci. 82:2611-2615.

2. ARÉCHIGA-FLÓREZ, C.F. 2000. Estrategias para incrementar la fertilidad de vacas lecheras expuestas a estrés calórico. In: Memorias Tópicos selectos en reproducción bovina. U.A.M.Z., U.A.Z. Zacatecas México. p.121-129.
3. ARÉCHIGA-FLOREZ, C.F.; HANSEN, P.J. 2003. Adverse impact of climate on reproductive processes of the bovine. Vet. Zacatecas. 2:89-107.

4. ARMSTRONG, D.V. 1994. Heat stress interaction with shade and cooling. J. Dairy Sci. 77:2044-2050.

5. ASHWORTH, C.J.; TOMA, L.M.; HUNTER, M.G. 2009. Nutritional effects on oocyte and embryo development in mammals: implications for reproductive efficiency and environmental sustainability. Phil. Trans. R. Soc. B 364:3351-3361.

6. BADINGA, L.; COLLIER, R.J.; THACHER, W.W.; WILCOX, C.J. 1985. Effects of climatic and management factors on conception rate of dairy cattle in subtropical environment. J. Dairy Sci. 68:78-85.

7. BADINGA, L.; THATCHERT, W.W.; DÍAZ, T.; DROST, M.; WOLFENSON, D. 1993. Effect of environmental heat stress on follicular development and steroidogenesis in lactating Holstein cows. Theriogenology. 39:797-810.

8. BAÑUELOS, R.; SILVA, J.M.; ARÉCHIGA, C.F. 2001. La función de las proteínas de choque calórico en la reproducción animal. Orinoquia 5(1):41-50.

9. BENYEI, B.; GASPARDY, A.; CSEH, S. 2003. Effect of the El Niño phenomenon on the ovarian responsiveness and embryo production in donor cows. Acta. Vet. Hung. 51:209-218.

10. BETANCOURT, K.; IBRAHIM, M.; VILLANUEVA, C.; VARGAS, B. 2005. Efecto de la cobertura arbórea sobre el comportamiento animal en fincas ganaderas de doble propósito en Matiguás, Matagalpa, Nicaragua. Livestock Research for Rural Development. 17, Article \#81.Disponible desde Internet en: http://www.lrrd.org//rrd17/7/ beta17081.htm (con acceso 21/04/10).

11. BIGGERS, B.G.; GEISERT, R.D.; WETTEMAN, R.P.; BUCHANAN, D.S. 1987. Effect of heat stress on early embryonic development in the cow. J. Anim. Sci. 64:1512-1518.

12. BOEDIONO, A.; SUZUKI, T.; GODKE, R.A. 2003 Comparison of hybrid and purebred in vitro-derived cattle embryos during in vitro culture. Anim. Reprod. Sci. 78:1-11.

13. BREUEL, K.F.; LEWIS, P.E.; SCHRICK, F.N.; LISHMAN, A.W.; INSKEEP, E.K.; BUTCHER, R.L. 1993. Factors 
affecting fertility in the pospartum cow: role of the oocyte and follicle in conception rate. Biol. Reprod. 48:655-661.

14. BUFFINGTON, D.E.; COLAZO-AROCHO, A.; CANTON, G.H.; PITT, D.; THATCHER, W.W.; WILCOX, R.J. 1981. Black-globe-humidity index (BGHI as comfort equation for dairy cows). Trans. Am. Soc. Agric. Eng. 24(4):711-714.

15. BURROW, H.M. 2001. Variances and covariances between productive and adaptive traits and temperament in a composite breed of tropical beef cattle. Livest. Prod. Sci. 70:213-233.

16. CABALLERO, C.S.; OCAMPO, C.L.; SUMANO, L.H. 1995. $\mathrm{El}$ uso de la somatotropina recombinante ( $\mathrm{STBr})$ durante el estrés calórico en ganado bovino. Tec. Pec. Mex. 33(3):168-176.

17. CHEBEL, R.C.; SANTOS, J.E.; REYNOLDS, J.P.; CERRI, R.L.; JUCHEM, S.O.; OVERTON, M. 2004. Factors affecting conception rate after artificial insemination and pregnancy loss in lactating dairy cows. Anim. Reprod. Sci. 84:239-55.

18. COLE, J.A.; HANSEN, P.J. 1993. Effects of administration of recombinant bovine somatotropin on the responses of lactating and nonlactating cows to heat stress. JAVMA. 203(1):113-117.

19. COLLIER, R.J.; DOELGER, S.G.; HEAD, H.H.; THATCHER, W.W.; WILCOX, C.J. 1982. Effects of heat stress during pregnancy on maternal hormone concentrations, calf birth weight and pospartum milk yield of Holstein cows. J. Anim. Sci. 54:309-319.

20. De CASTRO e PAULA, L.A.; ANDRZEJEWSKI, J.; JULIAN, D.; SPICER, L.J.; HANSEN, P.J. 2008 Oxygen and steroid concentrations in preovulatory follicles of lactating dairy cows exposed to acute heat stress. Theriogenology. 69: 805-813.

21. De DIOS VALLEJO, O. 2000. Ecofisiología de los bovinos en sistemas de producción del trópico húmedo. México, Universidad Juárez Autónoma de Tabasco; p.28-59.

22. DUNLAP, S.E.; VINCENT, C.K. 1971. Influence of postbreeding thermal stress on conception rate in beef cattle. J. Anim. Sci. 32:1216-1218.

23. FRASER, A.F.; BROOM, D.M. 1990. Farm animal behaviour and welfare. 3a ed. London (UK): Bailliére and Tindall. 437p.
24. GEISERT, R.D.; ZAVY, M.T.; BIGGERS, B.G. 1998. Effect of heat stress on conceptus and uterine secretions in the bovine. Theriogenology 29:1075-1082.

25. GIESECKE, W.H. 1985. The effect of stress on udder health of dairy cows. Onderstepoort. J. Vet. Res. 52:175-193.

26. GWAZDAUSKAS, F.C.; THATCHER, W.W.; KIDDY, C.A.; PAAPE, M.J.; WILCOX, C.J. 1981. Hormonal patterns during heat stress following PGF2a-tham salt induced luteal regression in heifers. Theriogenology. 16:271-285

27. GÓNGORA, A.; HERNÁNDEZ, A. 2006: Comportamiento sexual, duración del estro y del ciclo estral en novillas criollas sanmartineras y brahman del piedemonte llanero colombiano. Livestock Research for Rural Development. Vol. 18, Article \#1. Disponible desde Internet en: http://www.lrrd.org/lrrd18/1/gong18001. htm (con acceso 28/04/10).

28. HAMMOND, A.C.; OLSON, T.A.; CHASE, Jr. C.C.; BOWERS, E.J.; RANDEL, R.D.; MURPHY, C.N.; VOGT, D.W.; TEWOLDE, A. 1996. Heat tolerance in two tropically adapted Bos taurus breeds, Senepol and Romosinuano, compared with Brahman, Angus, and Hereford cattle in Florida. J. Anim. Sci. 74:295-303.

29. HANSEN, P.J.; ARÉCHIGA, C.F. 1999. Strategies for managing reproduction the heat stressed dairy cow. J. Anim. Sci. 77 (suppl. 2):36-50.

30. HANSEN, P.J. 2007. Exploitation of genetic and physiological determinants of embryonic resistance to elevated temperature to improve embryonic survival in dairy cattle during heat stress. Theriogenology 68S: S242-S249.

31. HEAD, H.H.H.; SCHICK, P.O.; WILCOX, C.J. 1981. Interrelationships of physical measures of placenta, cow and calf (Abstr.). J. Dairy. Sci. 64 (Suppl):161.

32. HERNÁNDEZ-CERÓN, J.; CHASE, Jr. C.C.; HANSEN, P.J. 2004. Differences in heat tolerance between preimplantation embryos from brahman, romosinuano, and angus breeds. J. Dairy Sci. 87:53-58.

33. HÖTZEL, M.J.; PIHEIRO MACHADO, L.C. FILIO. 2000. Estresse, factores estressores e ben estar na criaçáo animal. En: Santos, C.V.; y Mauro L. Vieira, M.L., eds. Anais de Etología Anais do XVIII Econtro Anual de Etologia, Florianápolis, Brasil. p.25. 
34. JASSIM, A.H.; RAY, D.E. 1986. Influence of season and environmental modifications on fertility of dairy cattle. Fourth Sci. Conf of Sci. Counc. Proc Agric. Res Iraq. 1(Part 3):1678-1694.

35. JIMÉNEZ, L.; HERNÁNDEZ, A. 1982. Morfología del alantocorion bovino entre los 27 y 88 días de gestación. Rev. ACOVEZ. 9(32):44-55.

36. JOHNSON, H.D.; KIBLER, H.H.; RAGSDALE, A.C.; BERRY, I.L.; SHANKLIN, M.D. 1961. Role of heat tolerance and production level in response of lactating Holstein to various temperature-humidity conditions (Abstract). J. Dairy Sci. 44:1191.

37. JU, J.C. 2005. Cellular responses of oocytes and embryos under thermal stress: hints to molecular signaling. Anim. Reprod. 2(2):79-90.

38. KORNMATITSUK, B.; CHANTARAPRATEEP, P.; KORNMATITSUK, S.; KINDAHL, H. 2008. Different Types of Postpartum Luteal Activity Affected by the Exposure of Heat Stress and Subsequent Reproductive Performance in Holstein Lactating Cows. Reproduction in Domestic Animals. 43(5):515-519.

39. KRININGER III, C.E.; BLOCK, J.; AL-KATANANI, Y.M.; CHASE, C.C.; HANSEN, P.J. 2003. Differences between Brahman and Holstein cows in response to estrus synchronization, superovulation and resistance of embryos to heat shock. Anim. Reprod. Sci. 78:13-24.

40. LEE, C.N. 1993. Environmental stress effects on bovine reproduction. Vet. Clin. North. Am. Food. Anim. Pract. 9:263-273.

41. LOTAN, E.; STURMAN, H.; WELLER, J.I.; EZRA, E. 1993. Effects of recombinant bovine somatotropin under conditions of high production and heat stress. J. Dairy Sci. 76:1394-1402.

42. MACKINNON, M.J.; MEYER, K.; D.D.; HETZEL, J.S. 1991. Genetic variation and covariation for growth, parasite resistance and heat tolerance in tropical cattle. Livest. Prod. Sci. 27:105-122.

43. MAUST, L.E.; MCDOWELL, R.E.; HOVEN, N.W. 1972. Effect of summer weather on performance of Holstein cows in three stages of lactation. J. Dairy Sci. 55:11331139.

44. MOBERG, G.P. 1975. Effects of environment and management stress on reproduction in the dairy cow. J. Dairy Sci. 59(9):1618-1624.
45. MOHAMMED, H.O. 1991. Transfer of immunoglobulins and survival of newborn calves. The Cornell Vet. 81(2):173-182.

46. MONTY, D.E.; RACOWSKY, C. 1987. In vitro evaluation of early embryo viability and development of summer heat-stressed superovulated dairy cows. Theriogenology. 28:451-465.

47. NEBEL, R.L.; JOBST, S.M.; DRANSFIELD, M.B.G.; PANDOLFI, S.M.; BAILEY, T.L. 1997. Use of radio frequency data communication system, HeatWatch, to describe behavioral estrus in dairy cattle (Abstract). J. Dairy Sci. 80(Suppl. 1):179.

48. OLSON, T.A.; HAMMOND, A.C.; CHASE Jr. C.C. 1997. Evidence for the existence of a major gene influencing hair length and heat tolerance in Senepol cattle (Abstract). J. Anim. Sci. 75 (Suppl. 1):147.

49. PATTON, A.P. 1994. The dairy cow in a hot environment: Productive and physiological changes and their effect on management. In: Memorias del primer curso integral sobre estrés en animales domésticos. F.M.V.Z. U.N.A.M. México. D.F.

50. PEGORER, M.F.; VASCONCELOS, J.L.M.; TRINCA, L.A.; HANSEN, P.J.; BARROS, C.M. 2007. Influence of sire and sire breed (Gyr vs. Holstein) on establishment of pregnancy and embryonic loss in lactating Holstein cows during summer heat stress. Theriogenology. 67:692-697.

51. PLASSE, D.; WARNICK, A.C.; KOGER, M. 1968. Reproductive behaviour of Bos indicus females in subtropical environment. I. Puberty and ovulation frequency in Brahman x British heifers. J. Anim. Sci. 27:94-100.

52. PLASSE, D.; WARNICK, A.C.; KOGER, M. 1970. Reproductive behaviour of Bos indicus females in subtropical environment .IV. Length of estrous cycle, duration of estrus, time of ovulation, fertilization and embryo survival in grade Brahman heifers. J. Anim. Sci. 30:63-72.

53. PLASSE, D. 1994. Factores que influyen en la eficiencia reproductiva de bovinos de carne en América Latina Tropical y estrategias para mejorarla In: Memorias Manejo de la reproducción bovina en condiciones tropicales. Cartagena de Indias, Colombia.

54. PUTNEY, D.J.; MALAYER, J.R.; GROSS, T.S.; THATCHER, W.W.; HANSEN, P.J.; DROST, M. 1988. Heat stress-induced alterations in the synthesis and secre- 
tion of proteins and prostaglandins by cultured bovine conceptus and uterine endometrium. Biol. Reprod. 39:717-728.

55. PUTNEY, D.J.; DROST, M.; THATCHER, W.W. 1989. Influence of summer heat stress on pregnancy rates of lactating dairy cattle following embryo transfer or artificial insemination. Theriogenology. 31:765-778.

56. REYNOLDS, L.P.; FERREL, C.L.; NIENABER, J.A. 1985. Effects of chronic environmental heat stress on blood flow and nutrient uptake of the gravid uterus and foetus. J. Agric. Sci. Camb. 104:289-297.

57. RIVAS, P.C.; RODRÍGUEZ-MÁRQUEZ, J.M.; HERNÁNDEZ, A. 2006. Número de vasos sanguíneos y expresión del factor de crecimiento del endotelio vascular y de la óxido nítrico sintasa endotelial e inducida en la alantoides ovina. Rev. Cient. FCV-LUZ. 16(4):347-352.

58. ROBERTSHAW, D. 1984. Heat loss of cattle. In: Yousef, M.K. ed. Stress Physiology in Livestock Vol 1. Basic Principles ed. CRC Press. p.55-66.

59. RODRÍGUEZ, J.; HERNÁNDEZ, A.; HIDALGO, G. 2005. Área vascular del alantocorion ovino: un posible indicador post-mortem de sobrevivencia embrionaria. Rev. Cient. FCV-LUZ. 15(1):14-19.

60. ROTH, Z.; ARAV, A.; BOR, A.; ZERON, Y.; WOLFENSON, D. 2001. Improvement of quality of oocytes collected in the autumn by enhanced removal of impaired follicles from previously heat-stressed cows. Reproduction. 122:737-744.

61. ROTH, Z.; HANSEN, P.J. 2004. Involvement of apoptosis in disruption of oocyte competence by heat shock in cattle. Biol. Reprod. 71:1898-1906.

62. ROTH, Z.; HANSEN, P.J. 2005. Disruption of nuclear maturation and rearrangement of cytoskeletal elements in bovine oocytes exposed to heat shock during maturation. Reproduction. 129:235-244.

63. RUTLEDGE, J.J. 2001. Use of embryo transfer and IVF to bypass effects of heat stress. Theriogenology. 55:105-111.

64. SHEARER, J.K. 1992. Factors associated with concentrations of immunoglobulins in calostrum at the first milking post-calving. Prev. Vet. Med. 14:143-154.
65. SHEARER, J.K.; BRAY, D. 1995. Manteniendo la salud de la ubre y la calidad de la leche durante periodos calurosos. Hoard's Dairyman (Abstract).1(7):643.

66. SHEARER, J.K.; ELLIOT, J.B.; VARGAS, C. 1996. Raising replacement heifers in hot climates. In: Proceedings' of the International Conference on Livestock in the Tropics. Institute of Food and Agricultural Sciences University of Florida, Gainesville, Florida.

67. SHEHAB-EL-DEEN, M.A.M.M.; LEROY, J.L.M.R.; FADEL, M.S.; SALEHS, Y.A.; MAES, D.; VAN SOOM, A. 2010. Biochemical changes in the follicular fluid of the dominant follicle of high producing dairy cows exposed to heat stress early post-partum. Anim. Reprod. Sci. 117:189-200.

68. STOTT, G.H.; WIERSMA, F.; MENEFEE, B.E.; RADWANSKI, R. 1976. Influence of environmental of passive immunity in calves. J. Dairy Sci. 59: 1306-1311.

69. St-PIERRE, N.R.; COBANOV, B.; SCHNITKEY, G. 2003. Economic losses from heat stress by US livestock industries. J. Dairy Sci. 86:E52-E77.

70. THATCHER, W.W.; COLLIER, R.J. 1986. Effects of climate on bovine reproduction. In: Morrow, D.A. (ed). Current Veterinary Theriogenology. $2^{\text {nd }}$ Ed. Philadelphia. WB Saunders Co. p.301-309.

71. THATCHER, W.W.; SAVIO, J.; SCHMITT, E.; DROST, M. 1993. Regulación de la fertilidad a través del control del desarrollo folicular. In: Memorias de la Conferencia Internacional sobre Ganadería en los Trópicos. Universidad de Florida. Gainesville, Florida.

72. THATCHER, W.W. 1996. Enhancement of fertility in heatstressed cows. In: Proceedings of the International Conference on Livestock in the Tropics. Institute of food and agricultural sciences. University of Florida, Gainesville, Florida.

73. TORRES-JÚNIOR, J.R.S.; PIRES, FA.; de SÁ, W.F.; FERREIRA, de M.; VIANA, J.H.M.; CAMARGO, L.S.A.; RAMOS, A.A.; FOLHADELLA, I.M.; POLISSENI, J.; de FREITAS, C.; CLEMENTE, C.A.A.; de SÁ FILHO, M.F.; PAULA-LOPES, F.F.; BARUSELLI, P.S. 2008. Effect of maternal heat-stress on follicular growth and oocyte competence in Bos indicus cattle. Theriogenology. 69:155-166.

74. ULBERG, L.C.; BURFENING, P.J. 1967. Embryo death resulting from adverse environment on spermatozoa or ova. J. Anim. Sci. 26:571-577. 
75. WILSON, S.J.; KIRBY, C.J.; KOENIGSFELD, A.T.; KEISLER, D.H.; LUCY, M.C. 1998. Effects of controlled heat stress on ovarian function of dairy cattle. 2 . Heifers. J. Dairy Sci. 81:2132-2138.

76. WOLFENSON, D.; THATCHER, W.W.; BADINGA, L.; SAVIO, J.D.; MEIDAN, R.; LEW, B.J.; BRAW-TAL, R.; BERMAN, A. 1995. Effect of stress on follicular development during the estrous in lactating dairy cattle. Biol. Reprod. 52:1106-1113.

77. WOLFENSON, D.; LEW, B.J.; THATCHER, W.W.; GRABER, Y.; MEIDAN, R. 1997. Seasonal and acute heat stress effects on steroid production by dominant follicles in cows. Anim. Reprod. Sci. 47:9-19.

78. WOLFENSON, D.; ROTH, Z.; MEIDAN, R. 2000. Impaired reproduction in heat stressed cattle: basic and applied aspects. Anim. Reprod. Sci. 60-61:535-547.

79. YOUSEF, M.K. 1985. Heat production mechanism and regulation In: Yousef, K.J (ed) Stress physiology in Livestock Vol 2. Basic Principles. CRC. Press. p.175-194.

Recibido: Abril 12 de 2010

Aceptado: Septiembre 18 de 2010 